Results from the hematopoietic malignancy trial revealed that there were 481 hematologic malignancies in the inpatient cohort (319 lymphomas, 107 leukemias and 45 multiple myelomas) with an overall relative risk (RR) of 1.7. The results for the incident-RA cohort were strikingly similar: $R R=1.6$ for hematologic malignancy. In the TNF-antagonist cohort, there were 11 hematopoietic malignancies (9 lymphomas and 2 leukemias; $R R=2.1$ ). The marginally augmented risk of malignancy in the TNF-antagonist cohort might be a result of a higher disease activity in patients receiving TNF antagonists, which was not controlled for. The distribution of lymphoma subtypes was reviewed across the cohorts, and was found to be largely similar to previous reports of lymphomas in RA.

Results from the solid cancer study showed that in the inpatient cohort there were 3,379 cancers ( $R R=1.05$ for solid cancer). The risk was greater in men than in women because of the lower incidence of breast cancer in men. Colorectal cancer was reduced in this cohort, which might be due to use of nonsteroidal anti-inflammatory drugs; respiratory cancer increased, but this might be due to higher levels of smoking and higher levels of cancer detection in RA patients; and non-melanoma skin cancer was also more frequent. In the incident-RA cohort, there were 138 solid cancers $(R R=1.1)$. This cohort displayed a similar pattern of solid cancers to the inpatients cohort, but there was no reduction in risk of colorectal cancer and there was no increased risk of nonmelanoma skin cancer. In the TNF-antagonist cohort, there were 67 solid cancers $(R R=0.9)$. There was an increased frequency of nonmelanoma skin cancer but other specific sites of cancer were difficult to evaluate because of the low number of incident malignancies.

The data obtained from these trials should be regarded as interim results from an ongoing surveillance of these drugs. Indeed, continued monitoring of the long-term risk of TNF antagonists is needed to rule out a risk of cancers that have a longer induction time than that of the follow-up in these studies.

Rachel Murphy

Original articles Askling J et al. (2005) Haematopoietic malignancies in rheumatoid arthritis: lymphoma risk and characteristics after exposure to tumor necrosis factor antagonists. Ann Rheum Dis 64: 1414-1420

Askling J et al. (2005) Risks of solid cancers in patients with rheumatoid arthritis and after treatment with tumor necrosis factor antagonists. Ann Rheum Dis 64: 1421-1426

\section{Aerobic exercise reduces pain in older adults}

Extending their previous work demonstrating a reduction in pain in older adults who undertook regular intense exercise over a 6-year period, Bruce et al. have recently reported similar results after following the study group for a total of 14 years. As it was unknown whether long-term high-impact exercise such as running might increase pain, by causing fractures or soft-tissue injury, this study has helped to confirm the benefits of aerobic exercise in an elderly population.

The individuals studied included 492 members of an over-50s runners' association and 374 community-matched controls, randomly selected from a lipid-research study cohort comprising individuals of a similar age. To address the potential bias from self-selection and the presence of exercisers in the control group, all individuals in the study were also grouped into those who had ever run for exercise $(n=565)$ and those who had not $(n=301)$. Each person received an annual health-assessment questionnaire addressing exercise, health and demographic variables, including a pain assessment based on a visual analog scale $(0=$ no pain, $100=$ severe pain).

Over 14 years, individuals who had ever run had lower BMI and continued to exercise more vigorously than nonrunners, and members of the runners' association had pain levels approximately $25 \%$ lower than those of controls. Although fractures were more common in runners than nonrunners, after adjusting for sex, baseline BMI and study withdrawal, running was still associated with significantly reduced pain, contributing to a reduction in the morbidity generally associated with ageing.

Rebecca Doherty

Original article Bruce B et al. (2005) Aerobic exercise and its impact on musculoskeletal pain in older adults: a 14 year prospective, longitudinal study. Arthritis Res Ther 7 : R1263-R1270

\section{B-cell depletion with rituximab improves symptoms in treatment- refractory childhood SLE}

Childhood systemic lupus erythematosus (SLE) has an unpredictable natural history and 